\title{
ON A SYSTEM OF HAMILTON-JACOBI-BELLMAN INEQUALITIES ASSOCIATED TO A MINIMAX PROBLEM WITH ADDITIVE FINAL COST
}

\author{
SILVIA C. DI MARCO and ROBERTO L. V. GONZÁLEZ
}

\author{
Received 11 February 2003
}

\begin{abstract}
We study a minimax optimal control problem with finite horizon and additive final cost. After introducing an auxiliary problem, we analyze the dynamical programming principle (DPP) and we present a Hamilton-Jacobi-Bellman (HJB) system. We prove the existence and uniqueness of a viscosity solution for this system. This solution is the cost function of the auxiliary problem and it is possible to get the solution of the original problem in terms of this solution.
\end{abstract}

2000 Mathematics Subject Classification: 49L20, 49L25, 49K35, 49K15.

1. Introduction. The optimization of dynamic systems where the criterion is the maximum value of a function is a frequent problem in technology, economics, and industry. This problem appears, for example, when we attempt to minimize the maximum deviation of controlled trajectories with respect to a given "model" trajectory. Minimax problems differ from those usually considered in the optimal control literature where a cumulative cost is minimized. Since in some cases, minimax problems describe more appropriately decision problems arisen in controlled systems whose performance is evaluated with a unique scalar parameter, the minimax optimization has received considerable attention in recent publications (see, e.g., $[4,5,6,7,8,9,10,11,12,13,15,16$, $17,18,19,22])$. Furthermore, minimax problems are related to the design of robust controllers (see [14]).

In addition, from the academic point of view, the minimax optimal control problem is of interest in the area of game theory because minimax problems can be seen as a game (see [18]) where a player applies ordinary controls and the other one-using complete and privileged information-chooses a stopping time. Problems of this type lead to the treatment of nonlinear partial differential inequalities akin to those appearing in the obstacle problem (with obstacle given in explicit or implicit form, see [10]). To find solutions of these systems, we must consider generalized solutions-even discontinuous solutions-since commonly there do not exist classical solutions of such systems (see [2, 3]). The treatment of the infinite horizon problem also presents great analytical difficulties because the optimal cost is neither necessarily lower semicontinuous nor upper semicontinuous. Moreover, the 
optimal cost cannot be approximated with a sequence of finite horizon problems. Studies concerning these issues can be seen in $[17,19]$. Besides, it is also important to develop numerical methods to compute these solutions in an approximate way because in general it is not possible to find exact analytical solutions. Numerical methods to obtain approximated open-loop optimal controls are analyzed in [24] and for closed loop, see [8, 15].

Here, we analyze a minimax optimal control problem where the functional to be optimized not only depends on the maximum of a function along the complete trajectory of the system but also it takes into account (in an additive fashion) another function of the final state of the system.

For a problem with such a structure, a dynamical programming principle cannot be formulated merely in terms of the initial time and the initial state. To obtain a DPP, we introduce an auxiliary parameter which "remembers" the past maximum values (the use of a similar procedure can be seen in [4, 5]). Using this parameter, we present an auxiliary problem which gives the solution of the original problem when a particular value of the auxiliary parameter is chosen. For this second optimal control problem, we establish the associated dynamical programing principle (DPP) and a Hamilton-Jacobi-Bellman (HJB) equation. Finally, we prove that the optimal cost of the auxiliary problem is the unique solution of the associated HJB equation.

The paper is organized as follows. In Section 2, we present the optimization problem. In Section 3, we describe the auxiliary problem and its relation with the original one. We also establish there the dynamical programming equation. In Section 4, we give the HJB equation associated to the problem and we prove the uniqueness of the solution in the viscosity sense.

\section{The optimization problem}

2.1. Presentation of the problem. We consider a minimax optimal control problem with finite horizon and final cost. More specifically, the problem consists in minimizing the functional $J$,

$$
\begin{gathered}
J:[0, T] \times \mathbb{R}^{m} \times \mathscr{A} \longmapsto \mathbb{R}, \quad(t, x, \alpha(\cdot)) \longmapsto J(t, x, \alpha(\cdot)), \\
J(t, x, \alpha(\cdot))=\Psi\left(y_{\alpha}(T ; x, t)\right)+\underset{s \in[t, T)}{\operatorname{ess} \sup } f\left(s, y_{\alpha}(s ; x, t), \alpha(s)\right),
\end{gathered}
$$

where $y_{\alpha}(\cdot ; t, x)$ represents the state of a dynamic system which evolves from the pair $(t, x)$ according to the following differential equation:

$$
\begin{aligned}
y_{\alpha}^{\prime}(s ; t, x)= & g\left(s, y_{\alpha}(s ; t, x), \alpha(s)\right) \quad \text { a.e. } s \in[t, T], \\
& y_{\alpha}(t ; t, x)=x, \quad x \in \mathbb{R}^{m} .
\end{aligned}
$$

To simplify the notation, and whenever this simplification does not produce misunderstandings, we will write directly $y_{\alpha}(\cdot ; t, x)=y(\cdot)$. The set $\mathscr{A}=L^{\infty}((0$, $T) ; A$ ) is the set of admissible control policies and $A$ is the control set. 
The optimal cost function is given by

$$
u(t, x)=\inf _{\alpha(\cdot) \in \mathscr{A}} J(t, x, \alpha(\cdot))
$$

This problem is an extension of another one analyzed by Barron and Ishii [10] and by Di Marco and González [15, 18]. The problem with an additive final cost considered here can represent scenarios where the performance of the applied control is measured jointly (in an additive fashion) by the maximum of a function along the trajectory and by a function of the final state.

To give an example of this type of problems, we consider the following economic case.

\section{ELEMENTS OF THE PROBLEM.}

(1) The vector $y(t)$ of economic activities (manufacturing, services, etc.);

(2) $\operatorname{GDP}(y(T))$, gross domestic product at the end of the period $[0, T]$;

(3) $a(t)$, policy of resources assignments;

(4) $f(y(t), a(t))$, unemployment level.

We consider the following functional, which measures the effectiveness of the economic policy. The functional measures both the positive aspects (the GDP) and the negative ones (the unemployment level $f(y(t), a(t)))$ of the economic policy in the following form:

$$
J(a(\cdot))=\operatorname{GDP}(y(T)) / \max _{t \in[0, T]} f(y(t), a(t)) .
$$

The maximization of $J$ is equivalent to the maximization of the functional

$$
\log (\operatorname{GDP}(y(T)))-\max _{t \in[0, T]}(\log (f(y(t), a(t)))) .
$$

Hence, if we define

$$
\Psi(y(T))=-\log (\operatorname{GDP}(y(T)))
$$

the problem is equivalent to the minimization of the functional

$$
\Psi(y(T))+\max _{t \in[0, T]}(\log (f(y(t), a(t)))),
$$

and so we must deal with a problem of type (2.2).

As we will see, the new problem presents an additional difficulty because it is not possible to establish a DPP only in terms of the variables $(t, x)$. To avoid this difficulty, we introduce an auxiliary problem which generalizes the problem presented above and we establish the DPP corresponding to the auxiliary problem. We also present an HJB equation associated to the optimal cost. Finally, using a methodology similar to that one presented in [20, 23], we prove that the optimal cost is the unique solution of this HJB equation. 
2.2. General assumptions. Let $\operatorname{BUC}\left([0, T] \times \mathbb{R}^{m} \times A\right)$ be the set of bounded and uniformly continuous functions in $[0, T] \times \mathbb{R}^{m} \times A$. We assume that the following hypotheses hold:

$\left(\mathrm{H}_{1}\right) g:[0, T] \times \mathbb{R}^{m} \times A \mapsto \mathbb{R}^{m}, \mathfrak{g} \in \mathrm{BUC}\left([0, T] \times \mathbb{R}^{m} \times A\right)$, and

$$
\begin{aligned}
\|g(t, x, a)\| & \leq M_{g}, \\
\|g(t, x, a)-g(\hat{t}, \hat{x}, a)\| & \leq L_{g}(|t-\hat{t}|+\|x-\hat{x}\|),
\end{aligned}
$$

for all $t, \hat{t} \in[0, T]$, all $x, \hat{x} \in \mathbb{R}^{m}$, and all $a \in A ;$

$\left(\mathrm{H}_{2}\right) f:[0, T] \times \mathbb{R}^{m} \times A \mapsto \mathbb{R}, f \in \operatorname{BUC}\left([0, T] \times \mathbb{R}^{m} \times A\right)$, and

$$
\begin{gathered}
m_{f} \leq f(t, x, a) \leq M_{f}, \\
|f(t, x, a)-f(\hat{t}, \hat{x}, a)| \leq L_{f}(|t-\hat{t}|+\|x-\hat{x}\|),
\end{gathered}
$$

for all $t, \hat{t} \in[0, T]$, all $x, \hat{x} \in \mathbb{R}^{m}$, and all $a \in A ;$

$\left(\mathrm{H}_{3}\right) \Psi: \mathbb{R}^{m} \mapsto \mathbb{R}, \Psi \in \operatorname{BUC}\left(\mathbb{R}^{m}\right)$, and

$$
|\Psi(x)-\Psi(\hat{x})| \leq L \Psi|| x-\hat{x} \|, \quad \forall x, \hat{x} \in \mathbb{R}^{m}
$$

$\left(\mathrm{H}_{4}\right) \quad A$ is compact in $\mathbb{R}^{q}$.

Note 2.1. The above-stated hypotheses are not the minimal ones under which the principal results of this paper hold. In particular, the Lipschitz continuity of $f$ and $\Psi$ can be replaced by the uniform continuity. We have used $\left(\mathrm{H}_{1}\right),\left(\mathrm{H}_{2}\right),\left(\mathrm{H}_{3}\right)$, and $\left(\mathrm{H}_{4}\right)$ in order to simplify the proof of the central results.

3. Auxiliary problem. In the problem presented above, it is not possible to establish a DPP merely in terms of the variables $(t, x)$ because the optimal control policy generally depends not only on the current state $(t, x)$ but also on the past values of the trajectory. We see the following example.

EXAMPLE 3.1. We consider $(t, x) \in[0,1] \times[-2,2]$. The control set is $A=$ $[-0.5,0.5]$. The instantaneous cost $f$, the final cost $\Phi$, and the dynamic $g$ are given by

$$
\begin{aligned}
& f(t, x, a)=(t-0.5)^{-}+a^{-}, \quad \Phi(x)=x, \\
& g(t, x, a)=a(1.5-0.5|x|-0.5|| x|-1|) .
\end{aligned}
$$

Figure 3.1 shows the optimal trajectories corresponding to a couple of different initial points $(t, x)$.

So, the optimal control policy depends not only on the current state $(t, x)$ but also on the past values of the trajectory. 


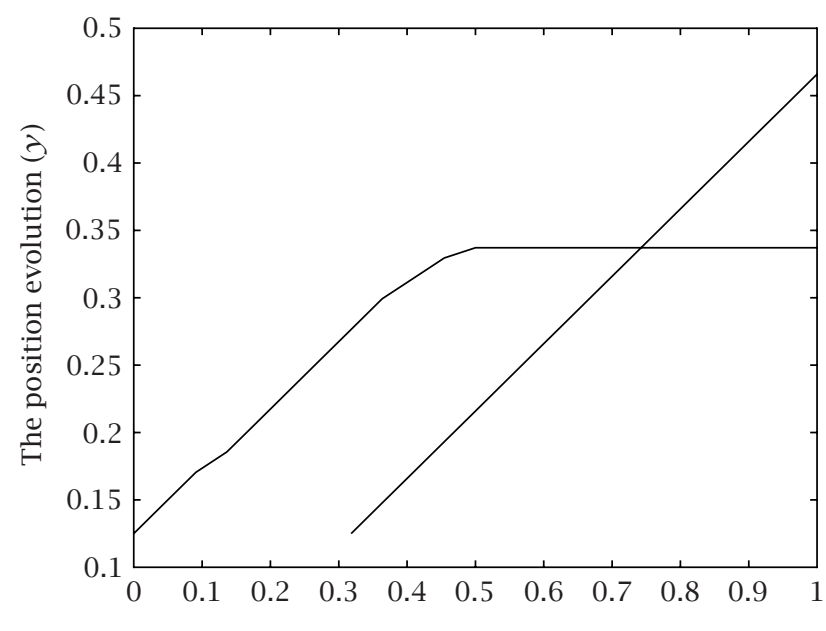

The time of evolution $(t)$

FIGURE 3.1

3.1. Auxiliary variable and problem reformulation. In order to develop the analytical and numerical treatment of the problem, we extend the state of the system introducing an auxiliary state $y_{m+1}(\cdot)$ (which is actually $y_{m+1}(\cdot):=$ $\left.y_{\alpha, m+1}(\cdot, t, x, \rho)\right)$. To simplify the notation, we will define

$$
h_{\alpha}(t, \tau):=\operatorname{esssup}_{s \in[t, \tau)} f(s, y(s), \alpha(s)), \quad \tau \in(t, T] .
$$

The auxiliary variable takes the following form, for all $\tau \in[t, T]$,

$$
y_{\alpha, m+1}(\tau, t, x, \rho)= \begin{cases}\rho, & \text { if } \tau=t, \\ \max \left(\rho, h_{\alpha}(t, \tau)\right), & \text { if } \tau \in(t, T] .\end{cases}
$$

NoTE 3.2. The additional variable $y_{m+1}$ "stores" the maximum value of the function $f$ from the initial time $t$ to the current time $\tau$ when its initial value is suitably chosen. More clearly, by considering $\rho \leq m_{f}$, from (3.3), it follows that

$$
y_{m+1}(T)=h_{\alpha}(t, T) \text {. }
$$

NoTE 3.3. When $\rho \in\left[m_{f}, M_{f}\right], y_{\alpha, m+1}(\tau, t, x, \rho) \in\left[m_{f}, M_{f}\right]$, for all $\tau \in$ $[t, T]$.

In this way, the functional cost becomes $J(t, x, \alpha(\cdot))=y_{m+1}(T)+\Psi(y(T))$ and the function $v$, defined as

$$
v(t, x, \rho)=\inf \left\{y_{\alpha, m+1}(T, t, x, \rho)+\Psi\left(y_{\alpha}(T, t, x)\right): \alpha(\cdot) \in \mathscr{A}\right\},
$$


where $t \in[0, T], x \in \mathbb{R}^{m}$, and $\rho \in\left[m_{f}, M_{f}\right]$, can be seen as the optimal cost of an ordinary optimal control problem, that is, a problem with pure final cost.

3.2. Properties of the optimal cost $v$. The following properties bring some relations between the optimal costs $u$ and $v$ of the original and auxiliary problems. They are almost evident and can be proved without difficulties using the definitions of the original and auxiliary problems described above. Here, we omit the complete proofs for the sake of brevity and we only sketch the lines of the argument.

(1) Let $m_{f}(t, x):=\min _{a \in A} f(t, x, a)$, then, for all $t \in[0, T]$ and for all $x \in \mathbb{R}^{m}$,

$$
u(t, x)=v\left(t, x, m_{f}(t, x)\right) .
$$

(2) We have $v\left(t, x, M_{f}\right)=M_{f}+\hat{u}(t, x)$, where $\hat{u}$ is the optimal cost of the optimal control problem where the functional to be minimized is $\widehat{J}(t, x, \alpha(\cdot))=$ $\Psi(y(T))$.

In this case $y_{m+1}(s)=M_{f}$, for all $s \in[t, T]$. Then, by replacing this equality in (3.5), we have

$$
v\left(t, x, M_{f}\right)=M_{f}+\inf _{\alpha(\cdot) \in \mathscr{A}} \Psi(y(T))=M_{f}+\hat{u}(t, x) .
$$

(3) If $\rho_{1}>\rho_{2}$, then

$$
v\left(t, x, \rho_{1}\right) \geq v\left(t, x, \rho_{2}\right)
$$

Let $\rho_{1}>\rho_{2}$, then

$$
\begin{aligned}
y_{\alpha, m+1}\left(T, t, x, \rho_{1}\right) & =\max \left\{\rho_{1}, h_{\alpha}(t, T)\right\} \\
& \geq \max \left\{\rho_{2}, h_{\alpha}(t, T)\right\}=y_{\alpha, m+1}\left(T, t, x, \rho_{2}\right) .
\end{aligned}
$$

By replacing this inequality in (3.5), it results that $v\left(t, x, \rho_{1}\right) \geq v\left(t, x, \rho_{2}\right)$.

(4) The optimal cost $v$ is bounded in $[0, T] \times \mathbb{R}^{m} \times\left[m_{f}, M_{f}\right]$.

This property follows from the definition of $v$ and the boundedness of $f$ and $\Psi$.

(5) The optimal cost $v$ is Lipschitz continuous with respect to the variables $t$, $x$, and $\rho$.

This property follows from the hypotheses verified by $f, g$, and $\Psi$. The proof can be obtained essentially with the techniques used in [21].

NOTE 3.4. As we have pointed out in Note 2.1, it is possible to consider $f$ and $\Psi$ uniformly continuous with respect to their variables. It also results that $v$ is continuous with respect to the variables $t, x$, and $\rho$ and similar results can be obtained. 
3.3. Dynamical programming equation. In this new problem with the state augmented by the variable $\rho$, the dynamical programming equation is given by

$$
v(t, x, \rho)=\inf _{\alpha(\cdot) \in L^{\infty}((t, s) ; A)} v\left(s, y(s), y_{m+1}(s)\right), \quad \forall s \in(t, T) .
$$

We also have the final condition

$$
v(T, x, \rho)=\max \left\{\rho, \min _{a \in A} f(T, x, a)\right\}+\Psi(x) .
$$

Both relations are almost evident and can be proved without difficulties using the classical argument of dynamic programming and the definition of the auxiliary problem described above. Here, we omit the complete proofs for the sake of brevity.

\section{Hamilton-Jacobi-Bellman equation}

4.1. Preliminaries. We define

$$
\begin{gathered}
H_{\rho}(t, x, \rho, \nabla v)=\frac{\partial v}{\partial \rho}(t, x, \rho), \\
H(t, x, \rho, \nabla v)=\inf \left\{\frac{\partial v}{\partial t}(t, x, \rho)+\frac{\partial v}{\partial x}(t, x, \rho) g(t, x, a): f(t, x, a)<\rho\right\}, \\
H_{*}(t, x, \rho, \nabla v)=\inf \left\{\frac{\partial v}{\partial t}(t, x, \rho)+\frac{\partial v}{\partial x}(t, x, \rho) g(t, x, a): f(t, x, a) \leq \rho\right\} .
\end{gathered}
$$

Taking into account the dynamical programming equation, we obtain the following differential formulation. Strictly, it takes the following form:

$$
\begin{aligned}
& \min \left\{H_{\rho}(t, x, \rho, \nabla v), H(t, x, \rho, \nabla v)\right\} \geq 0, \\
& \min \left\{H_{\rho}(t, x, \rho, \nabla v), H_{*}(t, x, \rho, \nabla v)\right\} \leq 0
\end{aligned}
$$

with the final condition

$$
v(T, x, \rho)=\max \left\{\rho, \min _{a \in A} f(T, x, a)\right\}+\Psi(x),
$$

for all $(x, \rho) \in \mathbb{R}^{m} \times\left[m_{f}, M_{f}\right]$, and the boundary condition

$$
v\left(t, x, M_{f}\right)=M_{f}+\hat{u}(t, x),
$$

for all $(t, x) \in[0, T] \times \mathbb{R}^{m}$, where $\hat{u}$ is the optimal cost of the optimal control problem where the functional to be minimized is $\widehat{J}(t, x, \alpha(\cdot))=\Psi(y(T))$.

NoTE 4.1. It is clear that $H \geq H_{*}$. It can easily be proved that $H_{*}$ is the lower semicontinuous envelope of $H$, that is,

$$
\begin{aligned}
H_{*}(t, x, \rho, q)=\liminf _{\varepsilon \rightarrow 0}\left\{H\left(t^{\prime}, x^{\prime}, \rho^{\prime}, q^{\prime}\right):\left|t-t^{\prime}\right|<\varepsilon,\right. \\
\left.|| x-x^{\prime}||<\varepsilon,\left|\rho-\rho^{\prime}\right|<\varepsilon,\left\|q-q^{\prime}\right\|<\varepsilon\right\} .
\end{aligned}
$$


NoTE 4.2. When $f \equiv 0$, we have the Mayer problem. In this case, $m_{f}=M_{f}=$ 0 and the solution of the problem is given by (3.7) (with $M_{f}=0$ ). So, $\rho \equiv 0$ and the function $\hat{u}(t, x)$ is obtained by solving the classical HJB equation

$$
\inf _{a \in A}\left\{\frac{\partial v}{\partial t}(t, x, 0)+\frac{\partial v}{\partial x}(t, x, 0) g(t, x, a)\right\}=0
$$

with final condition $v(T, x, 0)=\Psi(x)$, for all $x \in \mathbb{R}^{m}$.

NoTE 4.3. When $\Psi \equiv 0$, we have the optimal control problem treated by Barron and Ishii [10]. We define $\bar{\rho}(t, x)=\max \left\{\rho \in\left[m_{f}, M_{f}\right]: v(t, x, \rho)=u(t, x)\right\}$. Then, in case $\rho>\bar{\rho}(t, x)$, we have $v(t, x, \rho)=\rho$. In case $\rho \leq \bar{\rho}(t, x)$, it follows that $v(t, x, \rho)=u(t, x)$. For the value $\rho=u(t, x)$, it can be proved that the system (4.2) becomes

$$
\begin{aligned}
& \inf _{a \in A}\left\{\frac{\partial u}{\partial t}(t, x)+\frac{\partial u}{\partial x}(t, x) g(t, x, a): f(t, x, a)<u(t, x)\right\} \geq 0, \\
& \inf _{a \in A}\left\{\frac{\partial u}{\partial t}(t, x)+\frac{\partial u}{\partial x}(t, x) g(t, x, a): f(t, x, a) \leq u(t, x)\right\} \leq 0,
\end{aligned}
$$

which is the HJB equation presented by Barron and Ishii [10]. Obviously, the final condition is $u(T, x)=v\left(T, x, m_{f}(T, x)\right)=m_{f}(T, x)=\min _{a \in A} f(T, x, a)$.

4.2. Viscosity solution. We say that a function $v$ is a solution in the viscosity sense of system (4.2) when it is both a subsolution and a supersolution in the viscosity sense of (4.2). These concepts are defined as follows.

In order to simplify the writing we define $\Omega=(0, T) \times \mathbb{R}^{m} \times\left(m_{f}, M_{f}\right)$. We also give the following definitions.

(1) The function $w$ is a subsolution in the viscosity sense if

(i) $w$ is continuous on $\bar{\Omega}$;

(ii) $w$ is upper-bounded on $\bar{\Omega}$;

(iii) $w$ verifies the following final condition:

$$
w(T, x, \rho) \leq \max \left\{\rho, \min _{a \in A} f(T, x, a)\right\}+\Psi(x),
$$

for all $(x, \rho) \in \mathbb{R}^{m} \times\left[m_{f}, M_{f}\right]$;

(iv) $w$ verifies the following upper condition: $w\left(\cdot, \cdot, M_{f}\right)$ is a viscosity solution of

$$
H_{*}\left(t, x, M_{f}, \nabla w\right)=0,
$$

with final condition $w\left(T, x, M_{f}\right) \leq M_{f}+\Psi(x)$, for all $x \in \mathbb{R}^{m}$;

(v) given $(t, x, \rho) \in \Omega$ and $\phi \in C^{1}(\Omega)$ such that $\phi-w$ has a minimum in $(t, x, \rho)$ in a neighborhood $\mathcal{N}(t, x, \rho)$, it results that

$$
\min \left\{H_{\rho}(t, x, \rho, \nabla \phi), H(t, x, \rho, \nabla \phi)\right\} \geq 0
$$


(2) The function $z$ is a supersolution in the viscosity sense if

(i) $z$ is continuous on $\bar{\Omega}$;

(ii) $z$ is lower-bounded on $\bar{\Omega}$;

(iii) $z$ verifies the following final condition:

$$
z(T, x, \rho) \geq \max \left\{\rho, \min _{a \in A} f(T, x, a)\right\}+\Psi(x),
$$

for all $(x, \rho) \in \mathbb{R}^{m} \times\left[m_{f}, M_{f}\right]$;

(iv) $z$ verifies the following upper condition: $z\left(\cdot, \cdot, M_{f}\right)$ is a viscosity solution of

$$
H_{*}\left(t, x, M_{f}, \nabla z\right)=0
$$

with final condition $z\left(T, x, M_{f}\right) \geq M_{f}+\Psi(x)$, for all $x \in \mathbb{R}^{m}$;

(v) given $(t, x, \rho) \in \Omega$ and $\phi \in C^{1}(\Omega)$ such that $\phi-z$ has a maximum in $(t, x, \rho)$ in a neighborhood $\mathcal{N}(t, x, \rho)$, it results that

$$
\min \left\{H_{\rho}(t, x, \rho, \nabla \phi), H_{*}(t, x, \rho, \nabla \phi)\right\} \leq 0 .
$$

\subsection{The optimal cost as a viscosity solution}

THEOREM 4.4. The optimal cost $v$ is a solution in the viscosity sense of the system (4.2).

Proof. The final condition is trivially verified from (3.11) and in Section 3.2 we have seen that $v$ is continuous, bounded, and nondecreasing with respect to $\rho$.

From (3.3), for $\rho=M_{f}$, the variable $\rho$ remains constant and so, we are dealing with an ordinary optimal control problem. Then, with classical arguments (see $[1,20])$, we obtain that $v$ verifies the upper condition (4.9).

Now, it remains to prove the last condition of the subsolution's definition and the last one of the supersolution's definition.

First, we will prove that $v$ is a subsolution of (4.2).

Let $(t, x, \rho) \in \Omega$ and $\phi \in C^{1}(\Omega)$ such that $\phi-v$ has a minimum in $(t, x, \rho)$ in a neighborhood $\mathcal{N}(t, x, \rho)$.

Since $v$ is nondecreasing in its third variable, we obtain

$$
v(t, x, \rho) \leq v(t, x, \rho+\eta)
$$

By the minimality of $\phi-v$ at $(t, x, \rho)$, it follows that

$$
\phi(t, x, \rho+\eta)-\phi(t, x, \rho) \geq v(t, x, \rho+\eta)-v(t, x, \rho) \geq 0 .
$$

Dividing by $\eta$ and taking limit when $\eta$ goes to zero, we have

$$
\frac{\partial \phi}{\partial \rho}(t, x, \rho) \geq 0
$$


Then,

$$
H_{\rho}(t, x, \rho, \nabla \phi) \geq 0 \text {. }
$$

We prove that $H(t, x, \rho, \nabla \phi) \geq 0$. Let $a \in A$ such that $f(t, x, a)<\rho$ and let $\left\{\alpha_{n}\right\}$ be a control sequence such that $\alpha_{n}(s)=a$ for all $s \in\left[t, t+n^{-1}\right]$.

From (3.10), we have

$$
v(t, x, \rho) \leq v\left(t+n^{-1}, y_{n}\left(t+n^{-1}\right), \max \left\{\rho, \operatorname{essip}_{s \in\left[t, t+n^{-1}\right]} f\left(s, y_{n}(s), \alpha_{n}(s)\right)\right\}\right) \text {. }
$$

Since

$$
\lim _{n \rightarrow \infty} \operatorname{esssup}_{s \in\left[t, t+n^{-1}\right]} f\left(s, y_{n}(s), \alpha_{n}(s)\right)=f(t, x, a), \quad \forall n \geq n_{0},
$$

we get

$$
\max \left\{\rho, \operatorname{esssup}_{s \in\left[t, t+n^{-1}\right]} f\left(s, y_{n}(s), \alpha_{n}(s)\right)\right\}=\rho,
$$

and then, from (4.18), we obtain

$$
v(t, x, \rho) \leq v\left(t+n^{-1}, y_{n}\left(t+n^{-1}\right), \rho\right) .
$$

By the minimality of $\phi-v$ at $(t, x, \rho)$, it follows that

$$
\begin{aligned}
\phi(t & \left.+n^{-1}, y_{n}\left(t+n^{-1}\right), \rho\right)-\phi(t, x, \rho) \\
& \geq v\left(t+n^{-1}, y_{n}\left(t+n^{-1}\right), \rho\right)-v(t, x, \rho) \geq 0 .
\end{aligned}
$$

Since

$$
\lim _{n \rightarrow \infty} \frac{y_{n}\left(t+n^{-1}\right)-x}{n^{-1}}=\lim _{n \rightarrow \infty} n \int_{t}^{t+n^{-1}} g\left(s, y(s), \alpha_{n}(s)\right) d s=g(t, x, a),
$$

dividing by $n^{-1}$ in (4.22) and taking limit when $n \rightarrow \infty$, we have

$$
\frac{\partial \phi}{\partial t}(t, x, \rho)+\frac{\partial \phi}{\partial x}(t, x, \rho) g(t, x, a) \geq 0 .
$$

Then, since $a$ is arbitrary, it results that

$$
H(t, x, \rho, \nabla \phi) \geq 0 \text {. }
$$

So, (4.17) and (4.25) imply that $v$ is subsolution.

Now, we will show that $v$ is a supersolution of (4.2).

Let $(t, x, \rho) \in \Omega$ and $\phi \in C^{1}(\Omega)$ such that $\phi-v$ has a maximum at $(t, x, \rho)$ in a neighborhood $\mathcal{N}(t, x, \rho)$. 
We will prove by reductio ad absurdum that

$$
\min \left\{H_{\rho}(t, x, \rho, \nabla \phi), H_{*}(t, x, \rho, \nabla \phi)\right\} \leq 0 ;
$$

so, we assume that there exists $\eta>0$ such that

$$
\min \left\{H_{\rho}(t, x, \rho, \nabla \phi), H_{*}(t, x, \rho, \nabla \phi)\right\} \geq \eta>0 .
$$

Let $\left\{\alpha_{n}\right\}$ be a minimizing control sequence such that

$$
v(t, x, \rho)+\frac{1}{n^{2}} \geq v\left(t+n^{-1}, y_{n}\left(t+n^{-1}\right), \max \left\{\rho, \operatorname{esssup}_{s \in\left[t, t+n^{-1}\right]} f\left(s, y_{n}(s), \alpha_{n}(s)\right)\right\}\right) .
$$

We define

$$
\widehat{f}=\lim _{n \rightarrow \infty} \operatorname{esssup}_{s \in\left[t, t+n^{-1}\right]} f\left(s, y_{n}(s), \alpha_{n}(s)\right) .
$$

We suppose that $\hat{f}>\rho$. Taking limit in (4.28), it follows that $v(t, x, \rho) \geq$ $v(t, x, \hat{f})$. By the monotony of $v$, it results that $v(t, x, \rho)=v(t, x, \hat{f})$ and $v(t, x, \rho)=v(t, x, \varrho)$ for all $\varrho \in[\rho, \hat{f}]$. This implies that

$$
0=v(t, x, \varrho)-v(t, x, \rho) \geq \phi(t, x, \varrho)-\phi(t, x, \rho)
$$

and then $(\partial \phi / \partial \rho)(t, x, \rho) \leq 0$. Inequality (4.27) implies that $(\partial \phi / \partial \rho)(t, x, \rho)=$ $H_{\rho}(t, x, \rho, \nabla \phi) \geq \eta$. In consequence, $\eta \leq 0$, which is absurd.

We suppose that $\hat{f} \leq \rho$. We will analyze the effect of this condition on the following relation (valid by virtue of (4.27)):

$$
H_{*}(t, x, \rho, \nabla \phi) \geq \eta>0 .
$$

As $\hat{f} \leq \rho$, then, for all $\varepsilon>0$, there exists $n_{\varepsilon}$ such that, for all $n \geq n_{\varepsilon}$,

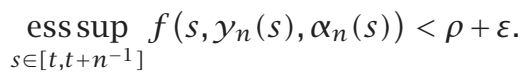

Eventually, by redefining the controls $\alpha_{n}(\cdot)$ in zero-measure sets, it is possible to affirm that, for all $n \geq n_{\varepsilon}$, for all $s \in\left[t, t+n^{-1}\right]$,

$$
f\left(s, y_{n}(s), \alpha_{n}(s)\right)<\rho+\varepsilon .
$$

Since $f$ is Lipschitz-continuous, there exists $n_{\varepsilon}$ such that, for all $n \geq n_{\varepsilon}$ and for all $s \in\left[t, t+n^{-1}\right]$,

$$
f\left(t, x, \alpha_{n}(s)\right)<\rho+\varepsilon+L_{f}\left(1+M_{g}\right) n^{-1}<\rho+2 \varepsilon .
$$


We consider the set of controls $Z_{\varepsilon}$ given by

$$
Z_{\varepsilon}=\{a \in A: f(t, x, a) \leq \rho+2 \varepsilon\} .
$$

Then, for any $n \geq n_{\varepsilon}$ and $s \in\left[t, t+n^{-1}\right]$, it results that $\alpha_{n}(s) \in Z_{\varepsilon}$.

As $(t, x, \rho)$ is a maximum point for the function $\phi-v$, we have

$$
\begin{aligned}
\phi(t & \left.+n^{-1}, y_{n}\left(t+n^{-1}\right), \rho\right)-\phi(t, x, \rho) \\
& \leq v\left(t+n^{-1}, y_{n}\left(t+n^{-1}\right), \rho\right)-v(t, x, \rho) .
\end{aligned}
$$

Since $v$ is nondecreasing in " $\rho$," from (4.28), we get

$$
\begin{aligned}
& v\left(t+n^{-1}, y_{n}\left(t+n^{-1}\right), \rho\right) \\
& \quad \leq v\left(t+n^{-1}, y_{n}\left(t+n^{-1}\right), \max \left\{\rho, \operatorname{esssup}_{s \in\left[t, t+n^{-1}\right]} f\left(s, y_{n}(s), \alpha_{n}(s)\right)\right\}\right) \\
& \quad \leq v(t, x, \rho)+n^{-2},
\end{aligned}
$$

thus,

$$
\phi\left(t+n^{-1}, y_{n}\left(t+n^{-1}\right), \rho\right)-\phi(t, x, \rho) \leq n^{-2}
$$

It is possible to prove that there exists $\bar{g} \in \overline{\operatorname{Co}\left(g\left(t, x, Z_{\varepsilon}\right)\right)}$, where $\operatorname{Co}(E)$ is the convex hull of the set $E$ such that (eventually using a suitable subsequence)

$$
\lim _{n \rightarrow \infty} n \int_{t}^{t+n^{-1}} g\left(s, y_{n}(s), \alpha_{n}(s)\right) d s=\bar{g},
$$

and so, by virtue of (4.38), we get

$$
\begin{array}{r}
\lim _{n \rightarrow \infty} \frac{\phi\left(t+n^{-1}, y_{n}\left(t+n^{-1}\right), \rho\right)-\phi(t, x, \rho)}{n^{-1}} \\
=\frac{\partial \phi}{\partial t}(t, x, \rho)+\frac{\partial \phi}{\partial x}(t, x, \rho) \bar{g} \leq 0 .
\end{array}
$$

On the other hand, as $Z_{\varepsilon}$ is closed, we have

$$
\overline{\operatorname{Co}\left(g\left(t, x, Z_{\varepsilon}\right)\right)}=\operatorname{Co}\left(g\left(t, x, Z_{\varepsilon}\right)\right) .
$$

As $\bar{g} \in \operatorname{Co}\left(g\left(t, x, Z_{\varepsilon}\right)\right)$ for all $\varepsilon>0$, we get that $\bar{g} \in \operatorname{Co}\left(g\left(t, x, Z_{0}\right)\right)$. Then, there exists a probability measure $\mu(\cdot)$ with support in $Z_{0}$ such that

$$
\bar{g}=\int_{Z_{0}} g(t, x, a) d \mu(a) .
$$

From (4.31), if $a \in Z_{0}$, then

$$
\frac{\partial \phi}{\partial t}(t, x, \rho)+\frac{\partial \phi}{\partial x}(t, x, \rho) g(t, x, a) \geq \eta>0 .
$$


Using this inequality and the integral expression (4.42) in (4.40), we have

$$
\begin{aligned}
0 & \geq \frac{\partial \phi}{\partial t}(t, x, \rho)+\frac{\partial \phi}{\partial x}(t, x, \rho) \bar{g} \\
& =\int_{Z_{0}}\left(\frac{\partial \phi}{\partial t}(t, x, \rho)+\frac{\partial \phi}{\partial x}(t, x, \rho) g(t, x, a)\right) d \mu(a) \geq \eta
\end{aligned}
$$

This inequality contradicts the initial assumption (4.27) and so, we obtain by reductio ad absurdum that $v$ is a supersolution.

\subsection{Uniqueness of the viscosity solution}

THEOREM 4.5. There is a unique viscosity solution of system (4.2).

Proof. Let $w$ be a subsolution and $z$ a supersolution of (4.2).

We will prove by reductio ad absurdum that $w \leq z$.

In the case $m_{f}=M_{f}$, the result is obvious because in that case the conditions (4.8) and (4.9) verified by $w$ and the conditions (4.9) and (4.11) verified by $z$ imply that $w \leq z$ (the proof now follows classical arguments (see [1, 20]) and it is here omitted for the sake of brevity). So, we will consider only the case $m_{f}<M_{f}$.

We suppose that

$$
r:=\sup \{w(t, x, \rho)-z(t, x, \rho):(t, x, \rho) \in \Omega\}>0
$$

then, there exists $\left(t_{r}, x_{r}, \rho_{r}\right) \in \Omega$ such that

$$
w\left(t_{r}, x_{r}, \rho_{r}\right)-z\left(t_{r}, x_{r}, \rho_{r}\right)>\frac{r}{2}
$$

Let $M>0$ such that

$$
\begin{gathered}
w(t, x, \rho) \leq \frac{M}{2}, \quad \text { in }[0, T] \times \mathbb{R}^{m} \times\left[m_{f}, M_{f}\right], \\
z(t, x, \rho) \geq-\frac{M}{2}, \quad \text { in }[0, T] \times \mathbb{R}^{m} \times\left[m_{f}, M_{f}\right] .
\end{gathered}
$$

For each $\epsilon>0$ and $\gamma>0$, we consider the hump function

$$
\begin{aligned}
\Phi(s, y, \zeta, t, x, \rho)= & -\frac{|t-s|^{2}}{\epsilon^{2}}-\frac{\|x-y\|^{2}}{\epsilon^{2}}-\frac{|\rho-\zeta+\sqrt{\epsilon}|^{2}}{\epsilon^{2}} \\
& -\sigma\left(\frac{T}{t}-1\right)-\sigma\left(\frac{M_{f}-m_{f}}{\rho-m_{f}}-1\right) \\
& -\frac{r}{8}\left(1-\frac{t}{T}\right)-2 M \xi\left(\gamma\left\|x-x_{r}\right\|\right) .
\end{aligned}
$$


The elements of $\Phi$ have the following properties:

(1) $\xi(\cdot) \in C^{1}(\mathbb{R})$ verifies

$$
\xi(\tau)= \begin{cases}0, & \forall \tau \in[0,1] \\ 1, & \forall|\tau| \geq 3\end{cases}
$$

for $\left|\xi^{\prime}(\tau)\right| \leq 1$, for all $\tau \in \mathbb{R}$;

(2) $\sigma<\min \left\{r t_{r} /\left(8\left(T-t_{r}\right)\right), r\left(\rho_{r}-m_{f}\right) /\left(8\left(M_{f}-m_{f}\right)\right)\right\}$.

We define the function

$$
\phi(s, y, \zeta, t, x, \rho)=w(s, y, \zeta)-z(t, x, \rho)+\Phi(s, y, \zeta, t, x, \rho)
$$

As $w$ is a subsolution, it must verify $H_{\rho}(t, x, \rho, \nabla w) \geq 0$. This condition implies that, for all $\rho \in\left[m_{f}, M_{f}\right)$ and for all $\varrho>0$ such that $\rho+\varrho \leq M_{f}$, we have $w(s, y, \rho+\varrho) \geq w(s, y, \rho)$ (the proof of this intuitive property is essentially contained in [23] and it is here omitted). Since $\rho_{r}<M_{f}$, we can suppose, without loss of generality, that $\epsilon$ is small enough to verify $\rho_{r}+\sqrt{\epsilon}<M_{f}$, and so

$$
w\left(t_{r}, x_{r}, \rho_{r}+\sqrt{\epsilon}\right)-w\left(t_{r}, x_{r}, \rho_{r}\right) \geq 0
$$

Now, taking into account that

$$
\frac{r}{8}\left(1-\frac{t_{r}}{T}\right) \leq \frac{r}{8}, \quad \sigma\left(\frac{T-t_{r}}{t_{r}}\right) \leq \frac{r}{8}, \quad \sigma\left(\frac{M_{f}-\rho_{r}}{\rho_{r}-m_{f}}\right) \leq \frac{r}{8}
$$

from (4.46), (4.48), (4.50), (4.51), and (4.52), we have

$$
\begin{aligned}
& \phi\left(t_{r}, x_{r}, \rho_{r}+\sqrt{\epsilon}, t_{r}, x_{r}, \rho_{r}\right) \\
& \quad \geq w\left(t_{r}, x_{r}, \rho_{r}+\sqrt{\epsilon}\right)-w\left(t_{r}, x_{r}, \rho_{r}\right)-\frac{3 r}{8}+\frac{r}{2} \geq \frac{r}{8}>0 .
\end{aligned}
$$

We consider a maximizing sequence $\left(s_{\mu}, y_{\mu}, \zeta_{\mu}, t_{\mu}, x_{\mu}, \rho_{\mu}\right)$ such that

$$
\lim _{\mu \rightarrow \infty} \phi\left(s_{\mu}, y_{\mu}, \zeta_{\mu}, t_{\mu}, x_{\mu}, \rho_{\mu}\right)=\sup _{\Omega \times \Omega} \phi(s, y, \zeta, t, x, \rho) .
$$

Let $\left(s_{\mu}, y_{\mu}, \zeta_{\mu}, t_{\mu}, x_{\mu}, \rho_{\mu}\right)$ be an element of this maximizing sequence. Therefore, for all $\mu \geq \mu_{0}$ suitably chosen, we have

$$
\phi\left(s_{\mu}, y_{\mu}, \zeta_{\mu}, t_{\mu}, x_{\mu}, \rho_{\mu}\right) \geq \phi\left(t_{r}, x_{r}, \rho_{r}+\sqrt{\epsilon}, t_{r}, x_{r}, \rho_{r}\right) \geq \frac{r}{8}>0 .
$$

Since (4.47) implies that

$$
w\left(s_{\mu}, y_{\mu}, \rho_{\mu}+\sqrt{\epsilon}\right)-z\left(t_{\mu}, x_{\mu}, \rho_{\mu}\right) \leq M
$$


from (4.48), (4.55), and (4.56), we get

$$
\begin{aligned}
\frac{\left|t_{\mu}-s_{\mu}\right|^{2}}{\epsilon^{2}} & +\frac{\left\|x_{\mu}-y_{\mu}\right\|^{2}}{\epsilon^{2}}+\frac{\left|\rho_{\mu}-\zeta_{\mu}-\sqrt{\epsilon}\right|^{2}}{\epsilon^{2}}+\sigma\left(\frac{T-t_{\mu}}{t_{\mu}}\right) \\
& +\sigma\left(\frac{M_{f}-\rho_{\mu}}{\rho_{\mu}-m_{f}}\right)+2 M \xi\left(\gamma\left\|x_{\mu}-x_{r}\right\|\right) \\
\leq & M-\frac{r}{8}<M .
\end{aligned}
$$

Then, inequality (4.57) and the properties of $\xi$ imply that

$$
\begin{gathered}
\frac{\sigma\left(M_{f}-m_{f}\right)}{M+\sigma}+m_{f}<\rho_{\mu}, \quad \frac{\sigma T}{M+\sigma}<t_{\mu}, \\
\left|t_{\mu}-s_{\mu}\right|<\sqrt{M} \epsilon, \quad\left\|x_{\mu}-y_{\mu}\right\|<\sqrt{M} \epsilon, \\
\left|\rho_{\mu}-\zeta_{\mu}+\sqrt{\epsilon}\right|<\sqrt{M} \epsilon, \quad\left\|x_{\mu}-x_{r}\right\| \leq \frac{3}{\gamma} .
\end{gathered}
$$

Moreover, in the case $m_{f}<M_{f}$, the conditions (4.8) and (4.9) verified by $w$ and the conditions (4.9) and (4.11) verified by $z$ imply that

$$
w\left(s, y, M_{f}\right) \leq z\left(s, y, M_{f}\right), \quad \forall(s, y) \in(0, T) \times \mathbb{R}^{m}
$$

(the proof is easy (see $[1,20]$ ) and it is here omitted).

We denote

$$
B_{r}=\left\{x \in \mathbb{R}^{m}:\left\|x-x_{r}\right\| \leq \frac{4}{\gamma}\right\}
$$

From (4.59) and the continuity of $w$ and $z$, we have that there exists $\delta_{r}>0$ such that if $x_{\mu} \in B_{r}, y_{\mu} \in B_{r}$, and

$$
\begin{gathered}
\left|t_{\mu}-s_{\mu}\right| \leq \delta_{r}, \quad|| x_{\mu}-y_{\mu} \| \leq \delta_{r}, \\
\zeta_{\mu} \geq M_{f}-\delta_{r}, \quad \rho_{\mu} \geq M_{f}-\delta_{r},
\end{gathered}
$$

then

$$
\phi\left(s_{\mu}, y_{\mu}, \zeta_{\mu}, t_{\mu}, x_{\mu}, \rho_{\mu}\right) \leq w\left(s_{\mu}, y_{\mu}, \zeta_{\mu}\right)-z\left(t_{\mu}, x_{\mu}, \rho_{\mu}\right) \leq \frac{r}{16}
$$

From (4.58), we have that, for $\epsilon$ small enough, conditions (4.61) are verified, $x_{\mu} \in B_{r}$, and $y_{\mu} \in B_{r}$. So, if (4.62) is verified, we get a contradiction between (4.55) and (4.63). In consequence, we get

$$
\zeta_{\mu}<M_{f}-\delta_{r} \quad \text { or } \quad \rho_{\mu}<M_{f}-\delta_{r} .
$$


Finally, this condition and (4.58) imply-again for $\epsilon$ small enough-that

$$
\zeta_{\mu}<M_{f}-\frac{\delta_{r}}{2}, \quad \rho_{\mu}<M_{f}-\frac{\delta_{r}}{2} .
$$

Consequently, $\left(s_{\mu}, y_{\mu}, \zeta_{\mu}, t_{\mu}, x_{\mu}, \rho_{\mu}\right)$ is a bounded sequence which verifies (4.58). Then, we can suppose, without loss of generality, that

$$
\left(s_{\mu}, y_{\mu}, \zeta_{\mu}, t_{\mu}, x_{\mu}, \rho_{\mu}\right) \longrightarrow\left(s_{\epsilon}, y_{\epsilon}, \zeta_{\epsilon}, t_{\epsilon}, x_{\epsilon}, \rho_{\epsilon}\right) \in \Omega \times \Omega
$$

In addition, from (4.58) and (4.65), we have

$$
\begin{aligned}
& \frac{\sigma\left(M_{f}-m_{f}\right)}{M}+m_{f} \leq \rho_{\epsilon}, \quad \frac{\sigma T}{M} \leq t_{\epsilon}, \\
& \left|t_{\epsilon}-s_{\epsilon}\right| \leq \sqrt{M} \epsilon, \quad\left\|x_{\epsilon}-y_{\epsilon}\right\| \leq \sqrt{M} \epsilon, \\
& \zeta_{\epsilon} \leq M_{f}-\frac{\delta_{r}}{2}, \quad \rho_{\epsilon} \leq M_{f}-\frac{\delta_{r}}{2}, \\
& \left\|x_{\epsilon}-x_{r}\right\| \leq \frac{3}{\gamma}, \quad\left|\rho_{\epsilon}-\zeta_{\epsilon}+\sqrt{\epsilon}\right| \leq \sqrt{M} \epsilon,
\end{aligned}
$$

and so,

$$
\left(s_{\epsilon}, y_{\epsilon}, \zeta_{\epsilon}, t_{\epsilon}, x_{\epsilon}, \rho_{\epsilon}\right) \in(0, T) \times B_{r} \times\left(m_{f}, M_{f}-\frac{\delta_{r}}{2}\right] \times(0, T) \times B_{r} \times\left(m_{f}, M_{f}-\frac{\delta_{r}}{2}\right] .
$$

From (4.54), we get

$$
\phi\left(s_{\epsilon}, y_{\epsilon}, \zeta_{\epsilon}, t_{\epsilon}, x_{\epsilon}, \rho_{\epsilon}\right)=\sup _{\Omega \times \Omega} \phi(s, y, \zeta, t, x, \rho)
$$

because $w$ and $z$ are continuous in $\Omega$, and then $w(s, y, \zeta)-z(t, x, \rho)$ is continuous in $\Omega \times \Omega$.

We define

$$
\begin{aligned}
& \psi:(0, T] \times B_{r} \times\left(m_{f}, M_{f}\right] \times(0, T] \times B_{r} \times\left(m_{f}, M_{f}\right] \longmapsto \mathbb{R}, \\
& \psi(s, y, \zeta, t, x, \rho)=w(s, y, \zeta)-z(t, x, \rho)-\sigma\left(\frac{M_{f}-m_{f}}{\rho-m_{f}}-1\right) \\
& -\sigma\left(\frac{T}{t}-1\right)-\frac{r}{8}\left(1-\frac{t}{T}\right)-2 M \xi\left(\gamma\left\|x-x_{r}\right\|\right), \\
& S_{\psi}=\sup \left\{\psi(t, x, \rho+\sqrt{\epsilon}, t, x, \rho):(t, x, \rho) \in(0, T] \times B_{r} \times\left(m_{f}, M_{f}-\frac{\delta_{r}}{2}\right]\right\} \text {, }
\end{aligned}
$$




$$
\begin{gathered}
\chi_{w, z}: \mathbb{R}_{0}^{+} \longmapsto \mathbb{R}_{0}^{+}, \\
\chi_{w, z}(\eta)=\sup \{\psi(s, y, \zeta, t, x, \rho) \\
-S_{\psi}:(t, x, \rho) \in[0, T] \times B_{r} \times\left(m_{f}, M_{f}-\frac{\delta_{r}}{2}\right], \\
(s, y, \zeta) \in[0, T] \times B_{r} \times\left(m_{f}, M_{f}-\frac{\delta_{r}}{2}\right], \\
|s-t|+\|y-x\|+|\zeta-\rho-\sqrt{\epsilon}| \leq \eta\} .
\end{gathered}
$$

It is obvious that $\chi_{w, z}$ is an increasing function verifying $\chi_{w, z}(0)=0$. In addition, from the continuity of $\psi$ in $(0, T] \times B_{r} \times\left(m_{f}, M_{f}\right] \times(0, T] \times B_{r} \times\left(m_{f}, M_{f}\right]$, we get $\lim _{\eta \rightarrow 0} x_{w, z}(\eta)=0$.

From the optimality of $\left(s_{\epsilon}, y_{\epsilon}, \zeta_{\epsilon}, t_{\epsilon}, x_{\epsilon}, \rho_{\epsilon}\right)$, we have for all $(t, x, \rho) \in(0, T] \times$ $B_{r} \times\left(m_{f}, M_{f}-\delta_{r} / 2\right]$ such that $\rho+\sqrt{\epsilon} \in\left(m_{f}, M_{f}\right]$,

$$
\begin{aligned}
\phi\left(s_{\epsilon}, y_{\epsilon}, \zeta_{\epsilon}, t_{\epsilon}, x_{\epsilon}, \rho_{\epsilon}\right) & \geq \phi(t, x, \rho+\sqrt{\epsilon}, t, x, \rho) \\
& =\psi(t, x, \rho+\sqrt{\epsilon}, t, x, \rho) .
\end{aligned}
$$

From this inequality and taking into account (4.50) and (4.70), we get

$$
\begin{aligned}
\frac{\left|t_{\epsilon}-s_{\epsilon}\right|^{2}}{\epsilon^{2}} & +\frac{\left\|x_{\epsilon}-y_{\epsilon}\right\|^{2}}{\epsilon^{2}}+\frac{\left|\rho_{\epsilon}-\zeta_{\epsilon}-\sqrt{\epsilon}\right|^{2}}{\epsilon^{2}} \\
& \leq \psi\left(s_{\epsilon}, y_{\epsilon}, \zeta_{\epsilon}, t_{\epsilon}, x_{\epsilon}, \rho_{\epsilon}\right)-\psi(t, x, \rho+\sqrt{\epsilon}, t, x, \rho) .
\end{aligned}
$$

From this inequality and (4.71), we get

$$
\frac{\left|t_{\epsilon}-s_{\epsilon}\right|^{2}}{\epsilon^{2}}+\frac{\| x_{\epsilon}-\left.y_{\epsilon}\right|^{2}}{\epsilon^{2}}+\frac{\left|\rho_{\epsilon}-\zeta_{\epsilon}-\sqrt{\epsilon}\right|^{2}}{\epsilon^{2}} \leq \psi\left(s_{\epsilon}, y_{\epsilon}, \zeta_{\epsilon}, t_{\epsilon}, x_{\epsilon}, \rho_{\epsilon}\right)-S_{\psi},
$$

and then, from (4.67) and (4.72),

$$
\frac{\left|t_{\epsilon}-s_{\epsilon}\right|^{2}}{\epsilon^{2}}+\frac{|| x_{\epsilon}-\left.y_{\epsilon}\right|^{2}}{\epsilon^{2}}+\frac{\left|\rho_{\epsilon}-\zeta_{\epsilon}-\sqrt{\epsilon}\right|^{2}}{\epsilon^{2}} \leq \chi_{w, z}(3 \sqrt{M} \epsilon) .
$$

From (4.76), we get

$$
\begin{array}{r}
\left|t_{\epsilon}-s_{\epsilon}\right| \leq \epsilon \sqrt{x_{w, z}(3 \sqrt{M} \epsilon),} \\
|| x_{\epsilon}-y_{\epsilon}|| \leq \epsilon \sqrt{x_{w, z}(3 \sqrt{M} \epsilon),} \\
\left|\rho_{\epsilon}-\zeta_{\epsilon}+\sqrt{\epsilon}\right| \leq \epsilon \sqrt{x_{w, z}(3 \sqrt{M} \epsilon)} .
\end{array}
$$

We define the function $\phi_{1} \in C^{1}\left((0, T) \times \mathbb{R}^{m} \times\left(m_{f}, M_{f}\right)\right)$ as follows:

$$
\phi_{1}(t, x, \rho)=w\left(s_{\epsilon}, y_{\epsilon}, \zeta_{\epsilon}\right)+\Phi\left(s_{\epsilon}, y_{\epsilon}, \zeta_{\epsilon}, t, x, \rho\right)
$$


From (4.50) and (4.69), $\phi_{1}-z$ has a maximum in $\left(t_{\epsilon}, x_{\epsilon}, \rho_{\epsilon}\right)$, then, as $z$ is a supersolution, it follows that

$$
\min \left(H_{\rho}\left(t_{\epsilon}, x_{\epsilon}, \rho_{\epsilon}, \nabla \phi_{1}\right), H_{*}\left(t_{\epsilon}, x_{\epsilon}, \rho_{\epsilon}, \nabla \phi_{1}\right)\right) \leq 0 .
$$

If

$$
\min \left(H_{\rho}\left(t_{\epsilon}, x_{\epsilon}, \rho_{\epsilon}, \nabla \phi_{1}\right), H_{*}\left(t_{\epsilon}, x_{\epsilon}, \rho_{\epsilon}, \nabla \phi_{1}\right)\right)=H_{\rho}\left(t_{\epsilon}, x_{\epsilon}, \rho_{\epsilon}, \nabla \phi_{1}\right),
$$

then

$$
H_{\rho}\left(t_{\epsilon}, x_{\epsilon}, \rho_{\epsilon}, \nabla \phi_{1}\right) \leq 0
$$

Since

$$
\begin{aligned}
\frac{\partial \phi_{1}}{\partial \rho}(t, x, \rho) & =\frac{\partial \Phi}{\partial \rho}\left(s_{\epsilon}, y_{\epsilon}, \zeta_{\epsilon}, t, x, \rho\right) \\
& =-2 \frac{\left(\rho-\zeta_{\epsilon}+\sqrt{\epsilon}\right)}{\epsilon^{2}}+\sigma \frac{\left(M_{f}-m_{f}\right)}{\left(\rho-m_{f}\right)^{2}}
\end{aligned}
$$

we have

$$
\begin{aligned}
H_{\rho}\left(t_{\epsilon}, x_{\epsilon}, \rho_{\epsilon}, \nabla \phi_{1}\right) & =\frac{\partial \phi_{1}}{\partial \rho}\left(t_{\epsilon}, x_{\epsilon}, \rho_{\epsilon}\right) \\
& =-2 \frac{\left(\rho_{\epsilon}-\zeta_{\epsilon}+\sqrt{\epsilon}\right)}{\epsilon^{2}}+\sigma \frac{\left(M_{f}-m_{f}\right)}{\left(\rho_{\epsilon}-m_{f}\right)^{2}}
\end{aligned}
$$

On the other hand, by defining $\phi_{2} \in C^{1}\left((0, T) \times \mathbb{R}^{m} \times\left(m_{f}, M_{f}\right)\right)$,

$$
\phi_{2}(s, y, \zeta)=z\left(t_{\epsilon}, x_{\epsilon}, \rho_{\epsilon}\right)-\Phi\left(s, y, \zeta, t_{\epsilon}, x_{\epsilon}, \rho_{\epsilon}\right),
$$

from (4.50) and (4.69), we have $\phi_{2}-w$ has a minimum in $\left(s_{\epsilon}, y_{\epsilon}, \zeta_{\epsilon}\right)$. As $w$ is a subsolution, we get

$$
H_{\rho}\left(s_{\epsilon}, y_{\epsilon}, \zeta_{\epsilon}, \nabla \phi_{2}\right) \geq 0
$$

Since

$$
\frac{\partial \phi_{2}}{\partial \zeta}(s, y, \zeta)=-\frac{\partial \Phi}{\partial \zeta}\left(s, y, \zeta, t_{\epsilon}, x_{\epsilon}, \rho_{\epsilon}\right)=-2 \frac{\left(\rho_{\epsilon}-\zeta+\sqrt{\epsilon}\right)}{\epsilon^{2}},
$$

we have

$$
H_{\rho}\left(s_{\epsilon}, y_{\epsilon}, \zeta_{\epsilon}, \nabla \phi_{2}\right)=\frac{\partial \phi_{2}}{\partial \zeta_{\epsilon}}\left(s_{\epsilon}, y_{\epsilon}, \zeta_{\epsilon}\right)=-2 \frac{\left(\rho_{\epsilon}-\zeta_{\epsilon}+\sqrt{\epsilon}\right)}{\epsilon^{2}} .
$$

Thus, from (4.85), we get $2\left(\rho_{\epsilon}-\zeta_{\epsilon}+\sqrt{\epsilon}\right) / \epsilon^{2} \leq 0$. Now, replacing this inequality in (4.83), we obtain

$$
H_{\rho}\left(t_{\epsilon}, x_{\epsilon}, \rho_{\epsilon}, \nabla \phi_{1}\right)=-2 \frac{\left(\rho_{\epsilon}-\zeta_{\epsilon}+\sqrt{\epsilon}\right)}{\epsilon^{2}}+\sigma \frac{\left(M_{f}-m_{f}\right)}{\left(\rho-m_{f}\right)^{2}}>0 .
$$


This inequality contradicts (4.81), then it is only possible that the following equality holds:

$$
\min \left(H_{\rho}\left(t_{\epsilon}, x_{\epsilon}, \rho_{\epsilon}, \nabla \phi_{1}\right), H_{*}\left(t_{\epsilon}, x_{\epsilon}, \rho_{\epsilon}, \nabla \phi_{1}\right)\right)=H_{*}\left(t_{\epsilon}, x_{\epsilon}, \rho_{\epsilon}, \nabla \phi_{1}\right) .
$$

In consequence, it must follow that

$$
H_{*}\left(t_{\epsilon}, x_{\epsilon}, \rho_{\epsilon}, \nabla \phi_{1}\right) \leq 0
$$

Let $\bar{a} \in A$ such that $f\left(t_{\epsilon}, x_{\epsilon}, \bar{a}\right) \leq \rho_{\epsilon}$ and

$$
\frac{\partial \phi_{1}}{\partial t}\left(t_{\epsilon}, x_{\epsilon}, \rho_{\epsilon}\right)+\frac{\partial \phi_{1}}{\partial x}\left(t_{\epsilon}, x_{\epsilon}, \rho_{\epsilon}\right) g\left(t_{\epsilon}, x_{\epsilon}, \bar{a}\right) \leq 0
$$

From (4.77), we get

$$
\begin{aligned}
f\left(s_{\epsilon}, y_{\epsilon}, \bar{a}\right) & \leq f\left(t_{\epsilon}, x_{\epsilon}, \bar{a}\right)+2 L_{f} \epsilon \sqrt{x_{w, z}(3 \sqrt{M} \epsilon)} \\
& \leq \rho_{\epsilon}+2 L_{f} \epsilon \sqrt{x_{w, z}(3 \sqrt{M} \epsilon)}
\end{aligned}
$$

Also, from (4.77), $\rho_{\epsilon} \leq \zeta_{\epsilon}+\epsilon \sqrt{\chi_{w, z}(3 \sqrt{M} \epsilon)}-\sqrt{\epsilon}$ and then (for $\epsilon$ small enough),

$$
\begin{aligned}
f\left(s_{\epsilon}, y_{\epsilon}, \bar{a}\right) & \leq \zeta_{\epsilon}+\epsilon \sqrt{\chi_{w, z}(3 \sqrt{M} \epsilon)}-\sqrt{\epsilon}+2 L_{f} \epsilon \sqrt{\chi_{w, z}(3 \sqrt{M} \epsilon)} \\
& \leq \zeta_{\epsilon}-\frac{\sqrt{\epsilon}}{2}<\zeta_{\epsilon}
\end{aligned}
$$

As $w$ is a subsolution, we have

$$
H\left(s_{\epsilon}, y_{\epsilon}, \zeta_{\epsilon}, \nabla \phi_{2}\right) \geq 0
$$

and so, by definition of $H$, we have

$$
\frac{\partial \phi_{2}}{\partial s}\left(s_{\epsilon}, y_{\epsilon}, \zeta_{\epsilon}\right)+\frac{\partial \phi_{2}}{\partial y}\left(s_{\epsilon}, y_{\epsilon}, \zeta_{\epsilon}\right) g\left(s_{\epsilon}, y_{\epsilon}, \bar{a}\right) \geq 0
$$

The derivatives of $\Phi$ are

$$
\begin{aligned}
& \frac{\partial \Phi}{\partial t}(s, y, \zeta, t, x, \rho)=-2 \frac{(t-s)}{\epsilon^{2}}+\sigma \frac{T}{t^{2}}+\frac{r}{8 T}, \\
& \frac{\partial \Phi}{\partial x}(s, y, \zeta, t, x, \rho)=-2 \frac{(x-y)}{\epsilon^{2}}-2 \gamma M \xi^{\prime}\left(\gamma\left\|x-x_{r}\right\|\right) \frac{\left(x-x_{r}\right)}{\left\|x-x_{r}\right\|}, \\
& \frac{\partial \Phi}{\partial s}(s, y, \zeta, t, x, \rho)=2 \frac{(t-s)}{\epsilon^{2}}, \quad \frac{\partial \Phi}{\partial y}(s, y, \zeta, t, x, \rho)=2 \frac{(x-y)}{\epsilon^{2}},
\end{aligned}
$$


and then,

$$
\begin{aligned}
\frac{\partial \phi_{2}}{\partial s}(s, y, \zeta) & =-\frac{\partial \Phi}{\partial s}\left(t_{\epsilon}, x_{\epsilon}, \rho_{\epsilon}, s, y, \zeta\right)=-2 \frac{\left(t_{\epsilon}-s\right)}{\epsilon^{2}} \\
\frac{\partial \phi_{2}}{\partial y}(s, y, \zeta) & =-\frac{\partial \Phi}{\partial y}\left(t_{\epsilon}, x_{\epsilon}, \rho_{\epsilon}, s, y, \zeta\right)=-2 \frac{\left(x_{\epsilon}-y\right)}{\epsilon^{2}} \\
\frac{\partial \phi_{1}}{\partial t}(t, x, \rho) & =\frac{\partial \Phi}{\partial t}\left(t, x, \rho, s_{\epsilon}, y_{\epsilon}, \zeta_{\epsilon}\right)=-2 \frac{\left(t-s_{\epsilon}\right)}{\epsilon^{2}}+\sigma \frac{T}{t^{2}}+\frac{r}{8 T} \\
\frac{\partial \phi_{1}}{\partial x}(t, x, \rho) & =\frac{\partial \Phi}{\partial x}\left(t, x, \rho, s_{\epsilon}, y_{\epsilon}, \zeta_{\epsilon}\right) \\
& =-2 \frac{\left(x-y_{\epsilon}\right)}{\epsilon^{2}}-2 \gamma M \xi^{\prime}\left(\gamma\left\|x-x_{r}\right\|\right) \frac{\left(x-x_{r}\right)}{\left\|x-x_{r}\right\|}
\end{aligned}
$$

Using these expressions in (4.91) and (4.95), we have

$$
\begin{aligned}
0<\frac{r}{8 T} \leq & -\sigma \frac{T}{t_{\epsilon}^{2}}+2 \frac{\left\langle x_{\epsilon}-y_{\epsilon}, g\left(t_{\epsilon}, x_{\epsilon}, \bar{a}\right)-g\left(s_{\epsilon}, y_{\epsilon}, \bar{a}\right)\right\rangle}{\epsilon^{2}} \\
& +2 \gamma M \xi^{\prime}\left(\gamma\left\|x_{\epsilon}-x_{r}\right\|\right) \frac{\left\langle x_{\epsilon}-x_{r}, g\left(t_{\epsilon}, x_{\epsilon}, \bar{a}\right)\right\rangle}{\left\|x_{\epsilon}-x_{r}\right\|} \\
\leq & \frac{2 L_{g}}{\epsilon^{2}}\left\|x_{\epsilon}-y_{\epsilon}\right\|\left(\left\|x_{\epsilon}-y_{\epsilon}\right\|+\left|t_{\epsilon}-s_{\epsilon}\right|\right)+2 \gamma M\left\|g\left(t_{\epsilon}, x_{\epsilon}, \bar{a}\right)\right\| \\
\leq & 4 L_{g} x_{w, z}(3 \sqrt{M} \epsilon)+2 \gamma M M_{g} .
\end{aligned}
$$

Letting successively $\epsilon$ and $\gamma$ go to zero, we have $r \leq 0$ which contradicts inequality (4.45). Then, $z(t, x, \rho) \geq w(t, x, \rho)$ for all $(t, x, \rho) \in \Omega$. Finally, taking into account that any solution is at the same time a subsolution and a supersolution, we obtain the uniqueness of solution.

5. Conclusion. In this paper, we have analyzed some issues concerning the viscosity solution of an HJB system associated to a minimax optimal control problem with finite horizon and additive final cost.

In the first step, we have introduced an auxiliary problem which generalizes the original one. It is possible to get the solution of the original problem in terms of the solution of the auxiliary problem.

For this auxiliary problem, a dynamical programming principle (DPP) holds. In relation with this DPP, we have presented an HJB system defined in terms of a discontinuous Hamiltonian and we have proved that the optimal cost of the auxiliary problem is the unique viscosity solution of this HJB system.

Although this problem could be seen as a particular (deterministic) case of those treated by Barles, Daher, and Romano [4], there are several differences between the results contained in [4] and those presented in this paper, among them are the following ones.

(1) We present direct proofs of the existence and uniqueness of solution without requiring the treatment of a sequence of $L^{p}$ problems. They are obtained through a different methodology based in control theory. 
(2) In our paper the HJB equation is deduced without the hypothesis $\operatorname{Im}(f)$ $=\left[m_{f}, M_{f}\right]$. So, our HJB equation is valid, in particular, for the cases where the controls take a finite number of values. In those special cases, the $L^{p}$ penalization technique used by Barles, Daher, and Romano does not allow deducing it.

(3) The HJB system here obtained is simpler than that one presented in [4] because no conditions are required at the lower boundary $(0, T) \times \mathbb{R}^{m} \times$ $\left\{m_{f}\right\}$.

\section{REFERENCES}

[1] M. Bardi and I. Capuzzo-Dolcetta, Optimal Control and Viscosity Solutions of Hamilton-Jacobi-Bellman Equations, Systems \& Control: Foundations \& Applications, Birkhäuser Boston, Massachusetts, 1997.

[2] G. Barles, Discontinuous viscosity solutions of first-order Hamilton-Jacobi equations: a guided visit, Nonlinear Anal. 20 (1993), no. 9, 1123-1134.

[3] __ Solutions de Viscosité des Équations de Hamilton-Jacobi [Viscosity Solutions of Hamilton-Jacobi Equations], Mathématiques \& Applications, vol. 17, Springer-Verlag, Paris, 1994 (French).

[4] G. Barles, Ch. Daher, and M. Romano, Optimal control on the $L^{\infty}$ norm of a diffusion process, SIAM J. Control Optim. 32 (1994), no. 3, 612-634.

[5]___ Convergence of numerical schemes for parabolic equations arising in finance theory, Math. Models Methods Appl. Sci. 5 (1995), no. 1, 125-143.

[6] E. N. Barron, Differential games with maximum cost, Nonlinear Anal. 14 (1990), no. 11, 971-989.

[7] _ The Pontryagin maximum principle for minimax problems of optimal control, Nonlinear Anal. 15 (1990), no. 12, 1155-1165.

[8] _ Averaging in Lagrange and minimax problems of optimal control, SIAM J. Control Optim. 31 (1993), no. 6, 1630-1652.

[9] _ Viscosity solutions and analysis in $L^{\infty}$, Nonlinear Analysis, Differential Equations and Control (Montreal, QC, 1998) (F. Clarke and R. J. Stern, eds.), NATO Sci. Ser. C Math. Phys. Sci., vol. 528, Kluwer Academic Publishers, Dordrecht, 1999, pp. 1-60.

[10] E. N. Barron and H. Ishii, The Bellman equation for minimizing the maximum cost, Nonlinear Anal. 13 (1989), no. 9, 1067-1090.

[11] E. N. Barron and R. Jensen, Relaxed minimax control, SIAM J. Control Optim. 33 (1995), no. 4, 1028-1039.

[12] E. N. Barron, R. Jensen, and J.-L. Menaldi, Optimal control and differential games with measures, Nonlinear Anal. 21 (1993), no. 4, 241-268.

[13] E. N. Barron and W. Liu, Semicontinuous solutions for Hamilton-Jacobi equations and the $L^{\infty}$-control problem, Appl. Math. Optim. 34 (1996), no. 3, 325-360.

[14] T. Başar and P. Bernhard, $H^{\infty}$-Optimal Control and Related Minimax Design Problems. A Dynamic Game Approach, Systems \& Control: Foundations \& Applications, Birkhäuser Boston, Massachusetts, 1991.

[15] S. C. Di Marco and R. L. V. González, Une procédure numérique pour la minimisation du coût maximum, C. R. Acad. Sci. Paris Sér. I Math. 321 (1995), no. 7, 869-873 (French).

[16] _ A finite state stochastic minimax optimal control problem with infinite horizon, Numerical Analysis and Its Applications (Rousse, 1996) (L. Vulkov, J. Waśniewski, and P. Yalamov, eds.), Lecture Notes in Comput. Sci., vol. 1196, Springer-Verlag, Berlin, 1997, pp. 134-141. 
[17] __ Supersolutions and subsolutions techniques in a minimax optimal control problem with infinite horizon, Indian J. Pure Appl. Math. 29 (1998), no. 10, 1083-1098.

[18] _ Minimax optimal control problems. Numerical analysis of the finite horizon case, M2AN Math. Model. Numer. Anal. 33 (1999), no. 1, 23-54.

[19] _ Relaxation of minimax optimal control problems with infinite horizon, J. Optim. Theory Appl. 101 (1999), no. 2, 285-306.

[20] W. H. Fleming and H. M. Soner, Controlled Markov Processes and Viscosity Solutions, Applications of Mathematics, vol. 25, Springer-Verlag, New York, 1993.

[21] A. Friedman, Differential Games, Pure and Applied Mathematics, vol. 25, WileyInterscience, New York, 1971.

[22] R. L. V. González and L. S. Aragone, A Bellman's equation for minimizing the maximum cost, Indian J. Pure Appl. Math. 31 (2000), no. 12, 1621-1632.

[23] R. L. V. González and E. Rofman, On unbounded solutions of Bellman's equation associated with optimal switching control problems with state constraints, Appl. Math. Optim. 31 (1995), no. 1, 1-17.

[24] E. Polak and L. M. He, Rate-preserving discretization strategies for semi-infinite programming and optimal control, SIAM J. Control Optim. 30 (1992), no. 3, 548-572.

Silvia C. Di Marco: CONICET, Instituto de Matematica Beppo Levi, FCEIA, Universidad Nacional de Rosario, Pellegrini 250, Rosario 2000, Argentina

E-mail address: dimarco@fceia.unr.edu.ar

Roberto L. V. González: CONICET, Instituto de Matematica Beppo Levi, FCEIA, Universidad Nacional de Rosario, Pellegrini 250, Rosario 2000, Argentina

E-mail address: r7vgonza@fceia.unr.edu.ar 


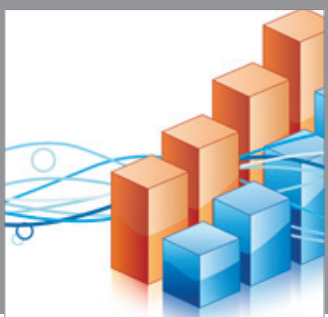

Advances in

Operations Research

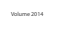

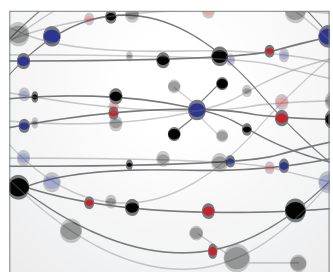

\section{The Scientific} World Journal
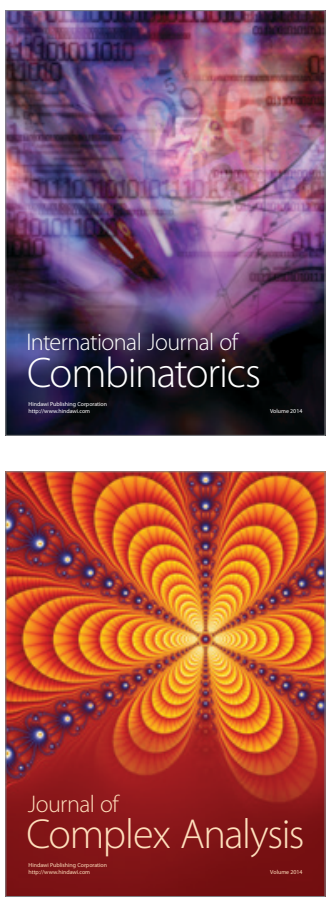

International Journal of

Mathematics and

Mathematical

Sciences
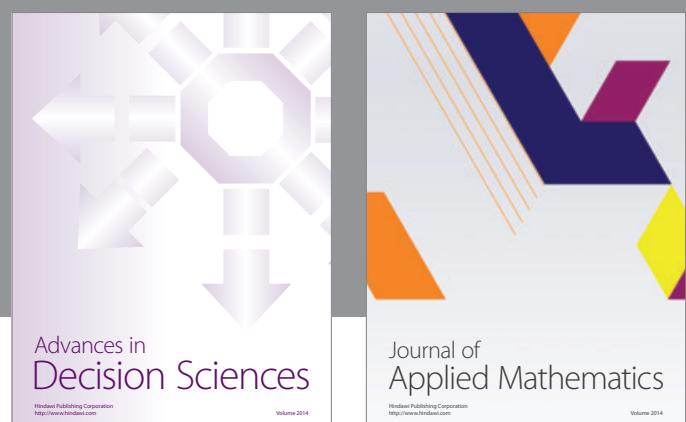

Journal of

Applied Mathematics
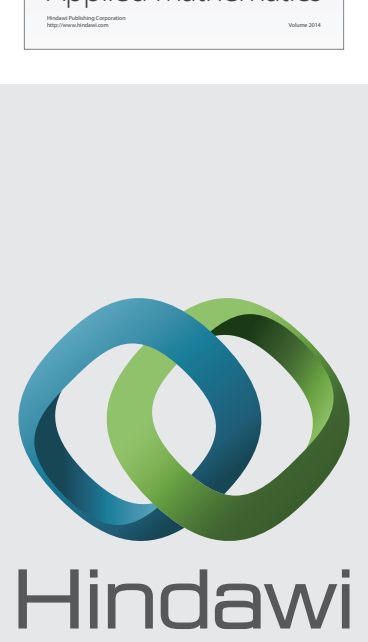

Submit your manuscripts at http://www.hindawi.com
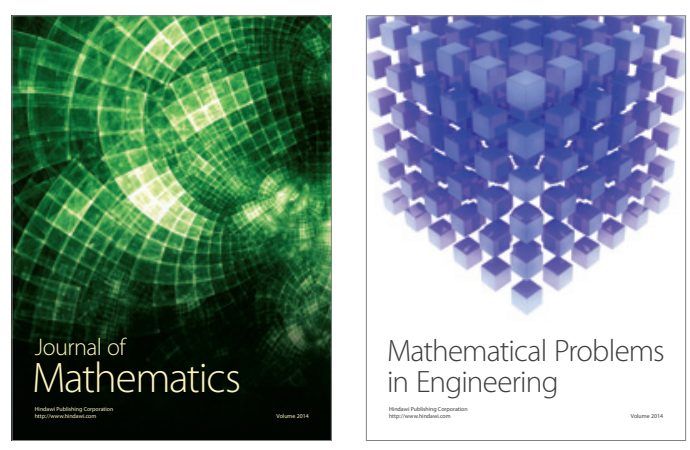

Mathematical Problems in Engineering
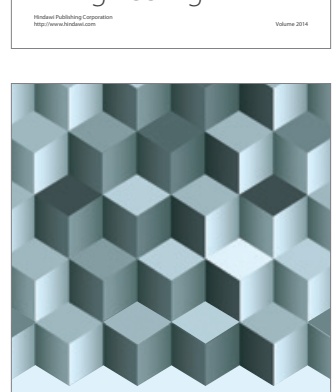

Journal of

Function Spaces
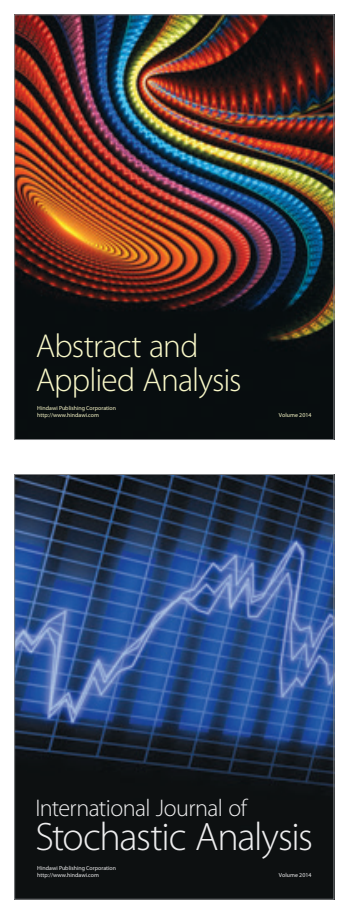

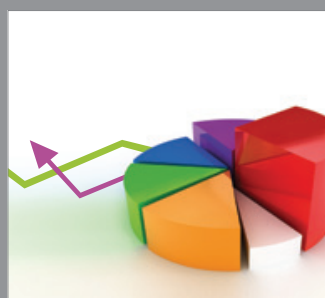

ournal of

Probability and Statistics

Promensencen
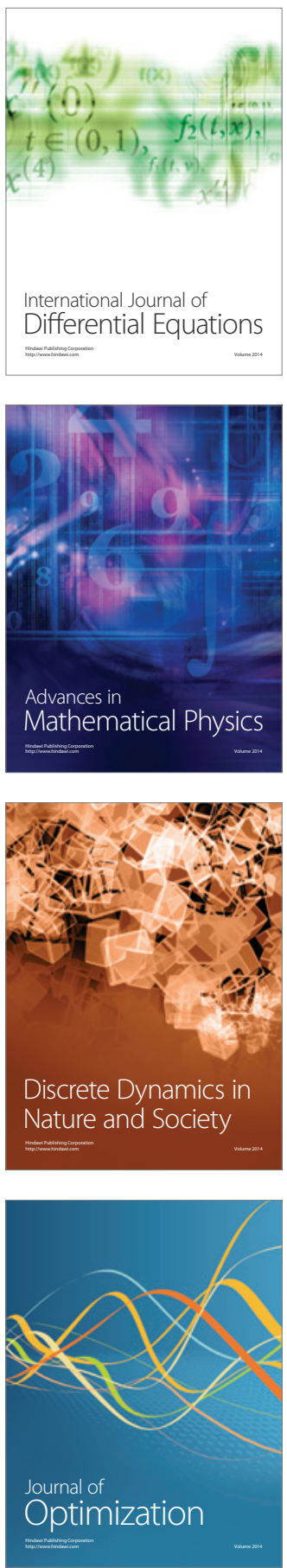